\title{
Norois
}

Environnement, aménagement, société

204 | 2007/3

Patrimoine, culture et construction identitaire dans les territoires ruraux

\section{La Rosière, incarnation et médiatrice d'une nouvelle ruralité : les villes-rosières de Gironde}

The May Queen as incarnation and mediator of a New Rurality: the case of urbanizing rural towns of Gironde

Marie-Dominique Ribereau-Gayon

\section{OpenEdition}

Journals

\section{Édition électronique}

URL : https://journals.openedition.org/norois/1440

DOI : $10.4000 /$ norois. 1440

ISBN : 978-2-7535-1552-9

ISSN : $1760-8546$

Éditeur

Presses universitaires de Rennes

Édition imprimée

Date de publication : 1 septembre 2007

Pagination : 53-65

ISBN : 978-2-7535-0533-9

ISSN : 0029-182X

Référence électronique

Marie-Dominique Ribereau-Gayon, «La Rosière, incarnation et médiatrice d'une nouvelle ruralité : les villes-rosières de Gironde », Norois [En ligne], 204 | 2007/3, mis en ligne le 01 septembre 2009, consulté le 14 janvier 2022. URL : http://journals.openedition.org/norois/1440 ; DOI : https://doi.org/ 10.4000 /norois. 1440 


\title{
LA RosiÈre, INCARNATION ET MÉDIATRICE D'UNE NOUVELLE RURALITÉ : LES VILLES-ROSIÈRES DE GIRONDE
}

\author{
Marie-Dominique Ribereau-Gayon \\ AMANDE-PAYMAR \\ (CEMAGREF - Bordeaux), \\ 50 avenue de Verdun - 33612 CESTAS cedex \\ marie-dominique.ribereau-gayon@laposte.net
}

\section{RÉSUMÉ}

Cet article montre comment des communes périurbaines de Gironde, déstabilisées par une urbanisation rapide, se saisissent d'une fête traditionnelle qui offrait une image désuète de la campagne pour produire une ruralité moderne, compatible avec une représentation urbaine de la campagne. Le temps de la fête, la Rosière - autrefois jeune fille vertueuse et aujourd'hui incarnation du civisme et d'un certain féminisme - sert de médiatrice dans la production de l'image d'une campagne idéale, esthétique, solidaire et qui fait consensus entre urbains et ruraux.

La Rosière - personne, fête et symbole - est mise à profit pour construire du lien social entre les divers groupes sociaux en présence et affirmer une continuité entre le passé et le présent du territoire. Incarnation de la permanence d'une identité rurale, la Rosière est désormais à la fois, objet patrimonial et facteur d'intégration sociale, voire de développement local.

MotS CLÉS : Bordeaux - Identité - Intégration - Patrimoine - Périurbanisation sociale - Rosière - Ruralité.

\section{ABSTRACT \\ The May Queen as incarnation and mediator of a New Rurality: the case of urbanizing rural towns of Gironde}

This paper is concerned with the response of some rural towns around Bordeaux to social perturbations due to massive urbanization. They chose to reactivate and reshape the outdated tradition of the election of a May Queen in order to produce a modern rurality compatible with urban representations of the countryside. For the duration of the festival, the May Queen - formerly a virtuous maiden and now a figure of public spiritedness and even feminism - mediates the construction of an ideal image of rurality, aesthetic and united, which suits both urban and rural dwellers.

The May Queen - as a person, a festival and a symbol-is ostensibly designed to induce social links between the various social groups in presence and to reassert the continuity between the territory's past and present. As a symbol of a preserved rural identity, the ceremony has become both a cultural heritage and a factor in social integration and even local development.

KEY WORDS : Bordeaux - Countryside - Heritage - May queen - Identity - Suburban Spaces - Social Integration. 
Comment une tradition rurale - l'élection d'une Rosière - si archaïque par son paternalisme, son puritanisme et son machisme qu'elle semblait inexorablement vouée à disparaître (Segalen, 1982) peut-elle participer aujourd'hui d'une ruralité moderne, compatible avec une représentation urbaine de la campagne et même un certain féminisme?

L'analyse de la redynamisation de cette coutume dans les communes de Gironde où elle subsiste - Grayan, Salleboeuf, Lesparre, La Brède, Créon, Pessac - apportera des éléments de réponse à cette question en dégageant le sens et les valeurs en jeu dans l'évolution. On s'attachera plus particulièrement aux trois dernières communes qui ont choisi de réactiver cette coutume afin de reconstituer le lien social menacé par la modification de leur structure sociale. L'évolution des modalités de sélection et de célébration de la Rosière illustre la manière dont les signes d'une ruralité en voie de disparition sont aujourd'hui travaillés pour construire la continuité identitaire de communes devenues ou devenant rurbaines, voire essentiellement urbaines. Après quelques données sur la situation des communes étudiées on présentera les évolutions de la coutume liées à l'urbanisation.

La démarche a inclus un recensement des villes-rosières, la collecte de données sur les communes et les modalités de la fête, une documentation sur les rites et fêtes dont celle de la Rosière est inspirée. En outre, pour les communes de Gironde, on a associé des entretiens semi-directifs auprès des acteurs directement impliqués, l'observation participante des fêtes, l'analyse d'archives municipales, d'articles de presse, de documents sur l'Internet, de gravures, photos et films privés et publics.

\section{Rosières, campagne et urbanisation}

Les données rassemblées sur les communes qui élisent une Rosière à l'heure actuelle montrent deux choses. D'une part, sur les 76 communes qui avaient une Rosière en 1999, plus de $72 \%$ sont des communes rurales de moins de 10000 habitants (tableau 1). D'autre part, c'est dans la tranche de population la plus élevée qu'on trouve les villes où la coutume a failli disparaître (Nanterre, Pessac, Châlon-sur-Saône) ou a disparu (toutes les communes de la banlieue parisienne, sauf Nanterre). Rosières et vie urbaine semblent donc incompatibles.

\begin{tabular}{|c|c|c|c|}
\hline Nombre d'habitants & Nombre de communes & & Communes de Gironde \\
\hline$<1000$ & 16 & \multirow{5}{*}{55} & Grayan \\
\hline 1000 à 2000 & 14 & & Sallebœuf \\
\hline 2000 à 3000 & 9 & & Créon \\
\hline 3000 à 5000 & 7 & & La Brède \\
\hline 5000 à 10000 & 9 & & Lesparre \\
\hline 10000 à 20000 & 12 & \multirow{4}{*}{21} & \\
\hline 20000 à 50000 & 4 & & \\
\hline 50000 à 100000 & 6 & & Pessac \\
\hline > à 100000 & 0 & & \\
\hline Total & 76 & & \\
\hline
\end{tabular}

Tableau 1 : Les Villes-Rosières françaises en 1999

French towns with a May queen in 1999

Paradoxalement, en Gironde, les communes ne font preuve d'un renouveau d'intérêt pour leur Rosière que lorsqu'elles sont atteintes par l'urbanisation (Lesparre et Sallebœuf en ce moment) et, parmi les six communes où la coutume subsiste, ce sont les plus urbanisées d'entre elles qui consacrent le plus d'énergie à la maintenir avec un succès certain. On est alors fondé à penser que la double équation : urbanisation = élimination de la ruralité = disparition des Rosières est, au moins partiellement, fausse. À y regarder de plus près c'est précisément parce que la coutume est 
en décalage avec le mode de pensée urbain qu’elle peut servir à affirmer la continuité identitaire et reconstruire du lien social.

\section{LES VILLES-ROSIÈRES DE GIRONDE}

La Brède, Créon et Pessac ont connu des augmentations de population remarquables par leur rapidité et leur ampleur : entre 1975 et 2004 la population de Créon a doublé et celle de La Brède a augmenté de $80 \%$, celle de Pessac a été multipliée par onze entre 1911 et 2004 avec des pics dans les années 1930, 1950 et 1960.

À l'époque de l'instauration de la fête (tableau 2) et aujourd'hui encore ce sont toutes des communes viticoles. Néanmoins, le prestige de leur production et sa place dans l'économie communale varient : seules les communes du Médoc (Lesparre, Grayan) vivent encore essentiellement de la viticulture et à Pessac, banlieue immédiate de Bordeaux, les châteaux de vin prestigieux voisinent avec les parcs industriels et technologiques et le campus universitaire.

\begin{tabular}{|l|l|l|l|l|}
\hline Commune & $\begin{array}{l}\text { Date du testament } \\
\text { fondateur }\end{array}$ & $\begin{array}{l}\text { Élection de la 1 } \\
\text { rosière }\end{array}$ & $\begin{array}{l}\text { Population lors de } \\
\text { la l } 1^{\text {re }} \text { éction }\end{array}$ & Population en 1999 \\
\hline La Brède & 1823 & 1824 & 1000 & 3128 \\
\hline Créon & 1892 & 1907 & 1000 & 2929 \\
\hline Grayan & 1893 & 1904 & n.d & 742 \\
\hline Pessac & 1896 & 1897 & 4396 & 56851 \\
\hline Sallebœuf & 1912 & 1913 & n.d & 1944 \\
\hline Lesparre & $1922(1947) 1$ & 1926 & n.d & 5044 \\
\hline
\end{tabular}

Tableau 2 : Les villes-rosières de Gironde Towns of Gironde with a May Queen

Le testament fondateur de Pessac calque les modalités d'élection et de célébration de la Rosière sur le modèle de La Brède (à l'exception de sa « touche aristocratique ») qui n'était pourtant pas le seul disponible en Gironde à l'époque. Les raisons de ce choix sont doubles. Tout d'abord, le testateur de La Brède conçoit la fête et la personne de la Rosière comme un hommage à l'esprit de Montesquieu, philosophe de renommée internationale dès cette époque et enfant du pays. Ce respectable référent donnait à la Rosière une image de marque que Pessac s'est appropriée pour ce qui a trait à la mise en forme de la ruralité bien plus que pour le fond philosophico-politique. L'autre raison est d'ordre socio-économique : la qualité des terroirs viticoles de La Brède et Pessac autant que les formes sociales (aristocratie du vin) et architecturales (châteaux) qui leur sont liées, les rapprochaient.

\section{DU MODÈLE ARISTOCRATIQUE AU MODÈLE POPULAIRE}

Mais, à la fin des années 1980, le désarroi engendré par les modifications démographiques incite Pessac à se tourner vers le modèle offert par Créon ${ }^{1}$. Bien que culturellement moins proche, Créon subissait également depuis le début des années 1970 une urbanisation rapide qui déstabilisait les relations sociales. En effet, les nouveaux venus - essentiellement urbains, cadres moyens et supérieurs - travaillent hors du bourg et sont donc peu présents sur le territoire communal de sorte que le spectre de la ville-dortoir se profilait dangereusement. À quelques années d'intervalle, La Brède s'est trouvée dans une situation analogue.

1. Créon est la «capitale » de l’Entre-deux-Mers pour les services scolaires, commerciaux, culturels et de santé. C'est ce qui a attiré la population nouvelle et explique son rayonnement. 
Rapidement, et malgré ses réticences personnelles vis-à-vis d'une tradition qu'il trouvait un peu ridicule, le maire de Créon décidait de moderniser la Rosière pour en faire un outil de restauration du lien social et de l'identité : «À Pessac ou à Talence on sait pas quand on en sort, quand on y rentre, c'est un défaut d'identité. [...] C'est tout le problème des banlieues, des villes satellites. [...] Je sentais que, ou bien on trouvait un terrain de rencontre, de convivialité, on reforgeait une identité, une personnalité et tous les prétextes étaient bons et la Rosière était un bon prétexte, ou bien on se faisait bouffer par Bordeaux » (M. C. ${ }^{2}$ ). Par conséquent, la ruralité des villes-rosières urbanisées ou rurbaines se définit moins, dès cette époque, par l'importance des activités agricoles que par le fait qu'elles sont moins urbaines que Bordeaux. C'est plus une question de degré que de nature, surtout pour Pessac.

La modernisation de la coutume porte sur plusieurs plans. Tout d'abord il s'agit de démocratiser la cérémonie et substituer la convivialité au formalisme guindé qui prévalait. Les maires et la Rosière ne s'adressaient pas la parole? Le maire de Créon danse dans la rue avec la Rosière, la fait rire et pousse la chansonnette lors du discours de couronnement. À La Brède, la Rosière est perchée sur un trône lui-même posé sur un haut catafalque? À Créon elle sera plus proche du public.

Il s'agit dans le même temps d'investir l'espace public pour associer plus largement la population - notamment celle qui ne fréquente pas l'église - et donner de l'ampleur à la cérémonie tout en rééquilibrant la partie religieuse et la partie laïque : dès la fin des années 1970 à Créon et depuis 1981 à Pessac, le couronnement a lieu hors de l'église, sur la place. De plus, au début des années 1980, le maire de Créon décide de rendre public le repas de la Rosière qui était jusque-là une affaire familiale privée. Aujourd'hui n'importe qui peut y participer en payant sa part. Depuis 2002, l'affluence ayant dépassé les capacités d'accueil du centre culturel, le repas a lieu sous un chapiteau planté sur un terrain légèrement excentré. Pour les animations, la totalité des lieux publics extérieurs et intérieurs sont investis soit simultanément soit successivement.

Il fallait encore agir sur deux autres plans : faire évoluer les critères de sélection de la jeune fille et renforcer l'inscription de la fête dans le temps. D’une journée à l'origine, la fête est passée à trois jours en 1995. Commençant le vendredi par un marché «à l'ancienne » et un repas sur la place centrale, elle se prolonge le samedi par la visite au cimetière, des animations, une fête foraine, des spectacles, un bal et se termine le dimanche par le parcours du cortège, la cérémonie religieuse à l'église, le discours du maire, le couronnement et la remise des prix et cadeaux sur la place. Le repas de la Rosière et un grand spectacle de clôture. Ce spectacle qui attire 4000 à 5000 spectateurs contribue à populariser la coutume hors de la commune; il met en œuvre des techniques de pointe qui donnent une image moderne de la fête. Multiplication des lieux publics, démocratisation des relations entre édiles et administrés, développement de la partie publique de la cérémonie, étalement dans le temps, tout cela a contribué à un renversement de perspective : on est passé d'une cérémonie formelle, à participation restreinte, à une fête populaire, largement installée dans l'espace et le temps de la commune et ouverte à un large public. Selon une ancienne Rosière, la fête actuelle est «plus agréable, c'est plus la fête et puis y a quand même une ambiance, une camaraderie qu'autrefois y avait pas » $\left(\mathrm{M}^{\text {me }} \mathrm{S} . \mathrm{V}^{3}{ }^{3}\right)$ Tel est maintenant, grosso modo, le processus d'évolution que suivent les autres villes-rosières de Gironde au fur et à mesure qu'elles se voient, à leur tour, confrontées à une modification importante de leur tissu social.

Si donc La Brède a fourni l'impulsion initiale, c'est aujourd'hui Créon qui est moteur, aux niveaux girondin et national, dans la théorisation de la fête. Pessac a connu une remarquable restauration après une période de quasi-faillite en changeant de modèle à temps. Les trois communes se sont auto-alimentées jusqu'en 2000 où la création d'une association nationale des villes-rosières, à l'initiative du maire de Créon, a permis de diffuser et enrichir ce qu'on pourrait appeler « le système Rosière ».

2. Maire de Créon de 1977 à 1995.

3. Ancienne Rosière de Créon; membre du Comité des Fêtes. 


\section{La Rosière, symbole de l'union entre l'individu et la collectivité}

Une Rosière est une jeune fille de famille modeste et répondant à des critères d'âge et de résidence, choisie pour sa vertu. Le jour de la cérémonie, des personnalités vont la chercher chez elle, en cortège. Elle porte une robe de mariée. Des échanges de fleurs ont lieu. Le cortège accompagne l'élue en musique jusqu'à l'église en s'arrêtant à divers endroits. Une messe est dite. Le maire expose au public les raisons du choix de la Rosière, la couronne de fleurs et lui remet un prix au nom de la collectivité. La fête est instaurée par le testament d'un donateur qui lègue des biens à cet effet. Un repas et des animations ont lieu. Ce schéma commun recouvre de multiples différences. Nous dégagerons leurs évolutions pour mettre en évidence les changements de valeur et d'image directement ou indirectement liées à l'urbanisation. Les trois communes étudiées ici ont également des Rosiers. Sans détailler, disons que la variabilité de leurs caractéristiques et l'obscurité de leur rôle mettent en évidence a contrario que la collectivité ne peut s'identifier qu'à une figure féminine (Agulhon, 1979).

\section{DE LA VERTU INDIVIDUELLE AUX VALEURS COLLECTIVES}

«Vertu » s'entend avant tout comme «virginité ». Mais, au XVI e siècle, la vertu inclus la pauvreté et, au XVIII ${ }^{\mathrm{e}}$ siècle, le respect filial. Au XIX ${ }^{\mathrm{e}}$ siècle, sa dimension religieuse s'affirme en associant virginité et respect des devoirs religieux puis, avec le développement d'une classe ouvrière, l'aptitude à supporter dignement la misère. Au cours du XIX ${ }^{\mathrm{e}}$ siècle, la vertu de la Rosière réside dans sa volonté d'échapper à sa condition sociale par le travail (Segalen, 1982). Aujourd'hui, sa vertu est explicitement, civique : l'engagement de l'élue dans des associations, des activités d'intérêt public, est devenu le critère essentiel.

Le mot vertu et toute référence à la virginité sont désormais, officiellement bannis. Toutefois, dans les représentations et les pratiques, la «valeur virginité » n’a pas complètement disparu. Ainsi, on peut élire une jeune fille qui a un compagnon mais « on en choisit quand même une qui ne court pas trop » (C. M. $\left.{ }^{4}\right)$. Il est également convenu tacitement que les petits amis restent discrets pendant la cérémonie. Navrés, les maires racontent aussi, les plaisanteries machistes rituelles : «Hé, tu l'as vérifié que la Rosière elle est méritante? » Celles-ci diminuent, depuis que les nouveaux habitants, et en particulier les femmes, siègent en nombre au conseil municipal. Ce changement ne résulte pas uniquement de l'urbanisation, mais s'inscrit dans l'évolution générale du regard porté sur les femmes. Néanmoins, l'arrivée d'une population porteuse de valeurs nouvelles a directement insufflé l'air du temps dans les communes rurales.

Parallèlement, l'évolution du critère d'âge, très indirectement liée à l'urbanisation, révèle un changement significatif de valence. En effet, la tranche d'âge (15-26 ans) définie dans les testaments était, tout logiquement, celle où se situaient les mariages à l'époque car le don à la Rosière était une dote pour son mariage. Actuellement, les Rosières sont pratiquement toutes élues l'année de leurs 18 ans. Alors que l'âge moyen au mariage n'a cessé de s'élever, l'âge moyen des Rosières n'a cessé de baisser, disjoignant ainsi de plus en plus élection et mariage. Choisir un individu qui se trouve au moment précis où il accède à la majorité sociale revient à mettre en exergue son indépendance sinon tout à fait encore son autonomie, saut qualitatif radical puisque l'élue n'est ni sous la dépendance des parents, ni sous celle d'un mari. En conséquence, seul le consentement de la jeune fille est sollicité de nos jours. Elle n'est pas dissociée de son environnement familial pour autant (une jeune fille, pompière bénévole, a été écartée parce que son père était, dit-on, alcoolique). Plus que son niveau social, c'est la capacité de la famille à participer à la fête qui compte. 
Marie-Dominique Ribereau-Gayon

\section{Du LIEU DE NAISSANCE ÀU LIEU DE RÉSIDENCE}

Tous les testaments stipulent que la Rosière doit être née dans la commune et appartenir à une «vieille » famille. Or, depuis le début des années 1970, aucune jeune fille n'est née dans les villes-rosières girondines, du fait de la progressive concentration des maternités vers les grandes villes. En outre, le maire de Pessac estime que $20 \%$ de la population de la commune change régulièrement. Toutes les communes se sont donc trouvées, à un moment ou à un autre, sans jeunes filles éligibles sauf à se livrer à quelques acrobaties mentales comme à La Brède, où on a élit une jeune fille née à Clermont-Ferrand, en s'accommodant du fait qu'elle descendait de la Rosière 1879. L'impossibilité soudaine de respecter ce critère de naissance et d'appartenance a été le déclencheur de toutes les évolutions. Pessac et Créon ont d'abord décidé que seraient éligibles les jeunes filles appartenant à une famille de souche puis, poussées plus avant par la mobilité démographique, celles dont les parents résidaient dans la commune au moment de leur naissance. Ces «bricolages » ne faisaient que reculer pour mieux sauter. Depuis $1990^{5}$ sont désormais éligibles les jeunes filles dont les parents résident dans la commune depuis plus de cinq ans, dix ans à La Brède toujours plus conservatrice. Par conséquent, la première étape de l'adaptation de la Rosière à l'urbanisation a consisté à passer d'un critère de naissance à un critère de résidence : c'est toute une idée de la permanence et de la relation au territoire de la commune qui se trouve ainsi bousculée, fragilisée par la réduction radicale de la profondeur du temps d'appartenance.

Cette première entorse officielle en a entraîné bien d'autres. En effet, le débat sur le maintien ou non du critère de résidence recouvrait en fait deux questions. La première - comment maintenir un éventail de choix? - était moins délicate que la seconde : faut-il ou non intégrer les nouveaux habitants et comment?

L'appartenance à une famille modeste et nombreuse, critère primordial à l'époque où le don à la Rosière représentait un avantage financier important ${ }^{6}$, est devenu très secondaire non seulement parce que les familles nombreuses se font rares, mais surtout parce qu'on privilégie maintenant les qualités personnelles de la jeune fille plutôt que la situation matérielle de sa famille. Le maire de Créon déclare chercher surtout à ce que la Rosière présente une image positive de la femme, quel que soit son milieu. N'oublions pas cependant que la plupart des nouveaux résidents sont relativement aisés par rapport à la population de souche. Par conséquent, maintenir un critère social rigide aurait empêché l’intégration des néo-habitants dans et par la fête.

\section{UNE FIGURE FÉMININE DE LA CITOYENNETÉ ET DE L’INTÉGRATION}

Si donc, le mariage effectif a quitté l'horizon, la forme de la cérémonie reste celle d'un mariage très classique. M. Segalen (1982) en avait déjà mis en évidence la dimension symbolique de mariage entre la collectivité et l'individu. Il est plus nouveau que cette valeur soit désormais explicitement intégrée. Cela a pour effet de réorienter les critères de sélection : « À partir de l'élection de M. C., on tombe vraiment dans les « Rosières citoyennes ». [...] On se prononce pratiquement plus sur la jeune fille elle-même mais sur son intégration dans la cité. [...] On recherche quand même quelqu'un qui a une pratique sociale, quelle qu'elle soit d'ailleurs, ça peut être artistique, sportive ou autre » (M. D.). À Pessac et La Brède, les jurés manifestent par leurs choix un intérêt particulier pour les jeunes filles qui ont des activités dans le domaine du social : actions humanitaires ou en faveur des handicapés, des enfants de milieux défavorisés, etc. (Despouys, 1996).

En matière d'intégration, la pratique sociale de la Rosière fonctionne à trois niveaux. Premièrement, au niveau individuel, sa participation à des activités collectives démontre sa capacité personnelle à s'intégrer dans un collectif et en fait un modèle pour les autres jeunes.

5. On officialisait ainsi une pratique en place depuis 1979.

6. En 1917, à Créon, la dotation de la Rosière représentait un an et demi de salaire d'une employée des postes. 
Deuxièmement, il s'agit au niveau de l'ensemble de la société, d'agir sur l'image sociale de la femme : le rôle public de la Rosière «fait passer » dans le collectif l'image positive et moderne de la femme qu'elle est censée incarner. « Le deuxième aspect de la modernité, je trouve, c'est le fait que les occasions dans lesquelles une jeune fille ou une jeune femme, je vais dire une jeune femme, joue un rôle social de leader dans une commune, je veux pas dire qu'elles sont rares mais, a priori, elles sont pas nombreuses, y a le sport et point final. Alors on peut dire que c'est une façon comme une autre de jouer un rôle, il est peut-être éphémère mais enfin, n'empêche, qu'à un moment donné il y a quelqu'un qui représente les femmes et qui joue un rôle social important » (M. D.). L'arrivée massive de couples urbains du tertiaire mettait, en effet, face à face deux images de la femme peu compatibles : disons, sommairement, d'une part les agricultrices, ancrées dans le territoire communal et sans autonomie financière et, d'autre part, des femmes à niveaux professionnel et culturel relativement élevés, mobiles et percevant des revenus de leur travail. La Rosière d'aujourd'hui conjoignant une image rurale avec une image moderne incarne la compatibilité des deux modèles et, par là, symbolise la possibilité d’intégration mutuelle des deux couches de population. Toutefois, si la réglementation de l'élection et le discours officiel ont achevé leur mue pour construire l'image d'une Rosière adulte, active, libre et citoyenne, on ne peut prétendre que toute la population de souche ait complètement intégré les changements, qui ne lui sont pas clairement présentés.

Enfin, à un troisième niveau, les modalités actuelles de sélection traduisent la volonté des communes d'œuvrer, grâce à la Rosière, à une intégration sociale plus large des nouveaux habitants : «[Parmi les Rosières] c'est pas par hasard que vous retrouvez des noms de deuxième génération et maintenant, je crois, que ce qui est recherché surtout, c'est bien abstrait, c'est la notion d'intégration. Comme on a dans cette commune un afflux de gens extérieurs, je crois que le principal critère qu'adoptent les gens, c'est l'intégration de la famille dans la vie locale, c'est-à-dire de ne pas choisir quelqu'un qui soit marginalisé » (M. D.). L'intégration des nouveaux habitants s'est d'abord limitée aux Français avant qu'on se préoccupe de l'intégration de la population d'origine immigrée qui est le défit actuel, nous le verrons.

\section{Rosière et société : reconstruire le lien social}

On attend donc explicitement que la coutume contribue directement au maintien et/ou à la restauration du lien social. Sur ce point, son efficacité se manifeste de nombreuses manières.

\section{RECONSTRUIRE LA RELATION ENTRE ÉLUS ET POPULATION}

En Gironde, la Rosière est choisie, suivant le testament, soit par le conseil municipal, soit par le bureau du comité des Fêtes, soit par un jury spécial. Dans tous les cas la sélection reposait sur le postulat que tout le monde connaissait tout le monde et que chaque juré pouvait donc avoir son mot à dire sur les jeunes filles. Or, à Pessac, dès les années 1950, la taille de la ville ne permettait plus aux jurés de connaître toutes les jeunes filles. Le risque était donc de ne voir élire que les «protégées » des jurés. En conséquence, depuis 1979, les candidates sont proposées par les comités de quartier et les associations qui rédigent une fiche anonyme présentant la situation familiale, les qualités de la jeune fille et surtout ses engagements dans des actions d'intérêt collectif. Par conséquent, du fait de l'augmentation de la population, la Rosière n'est plus l'élue d'un réseau d'inter-connaissance.

À La Brède et Créon, on fonctionne toujours selon le bouche-à-oreille. Le maire recense et présente les jeunes filles éligibles au jury, mais l'afflux de population rend le maintien du système problématique : «Plus ça va, moins on connaît les jeunes filles de maintenant [...]. J'arrive qu'on ne les connaît plus parce que y a tellement de gens nouveaux, tout ça » $\left(\mathrm{M}^{\mathrm{me}} \mathrm{O} \cdot{ }^{7}\right)$. Conscient

7. Créon, responsable du Comité des fêtes. 
du problème, chaque groupe met en place des procédés compensatoires sans qu’il y ait de plan concerté en la matière. Ces procédés varient selon la position par rapport à l'institution de la Rosière mais ont en commun de situer les stratégies dans le long terme. Ainsi, la responsable du comité des Fêtes de Créon qui recrute les enfants du cortège se fait communiquer par les institutrices les noms des élèves résidant dans la commune ${ }^{8}$ pour contacter leurs familles en prenant soin «qu'il y ait des nouveaux» $\left(\mathrm{M}^{\mathrm{me}} \mathrm{O}\right.$.). Cela permet aux mamans d'établir des relations dans le bourg et, à plus long terme, de placer les fillettes dans l'antichambre de l'élection. Le cortège des petits est un lieu où les nouveaux qui le souhaitent peuvent s'insérer dans un réseau d'interconnaissance que leur présence réifie. Les jeunes filles peuvent elles aussi mettre en place des stratégies pour se faire connaître : telle entre au Comité des Fêtes, telle autre fait un stage à la mairie ou chez un élu municipal et en profite pour faire savoir qu'elle aimerait bien être Rosière, telle autre se glisse dans le sillage de la Rosière et l'accompagne dans ses sorties officielles, etc. Enfin, le maire de Créon invite chaque année les nouveaux habitants à un pot de bienvenue au cours duquel il leur explique, notamment, la tradition de la Rosière, les invite à bien accueillir les jeunes qui font la collecte, les informe des diverses manières dont ils peuvent participer à la fête, et comment leurs filles pourront être élues. Mais, pour pouvoir jouer son rôle vis-à-vis du jury, il s'informe auprès des professeurs du collège sur les jeunes filles éligibles et les familles qu'il ne connaît pas. Les enseignants sont donc un nouveau et essentiel maillon dans la reconstruction, autour de la Rosière, d'une chaîne de relations entre les édiles et les nouveaux habitants.

\section{INTÉGRER PAR LE PARTAGE DE LA FÊTE}

Par ailleurs, un certain nombre de manifestations instaurées à partir des années 1980 à Créon, 1990 à Pessac, sont explicitement destinées à tisser du lien social. Leur succès montre que les nouveaux habitants se saisissent effectivement de ces occasions pour amorcer leur intégration dans la vie communale. Ainsi, à Créon, le comité des Fêtes de la Rosière a vu ses membres passer de 2 en 1988 à 56 en 1997 dont beaucoup de nouveaux habitants. De même, au banquet en plein air du vendredi soir, un millier de repas ont été servis en 1997, succès remarquable pour un petit bourg. Ce repas, où la Rosière est présente, mais sans rôle ni tenue spécifiques, permet aux nouveaux habitants, même ceux rebutés par l'archaïsme notoire de la Rosière, une première insertion, limitée et peu contraignante, dans la fête. De la même manière, la participation au repas du dimanche midi, s'accroît : une cinquantaine de personnes au début des années 1980, quelque 300 à la fin des années 1990, presque 400 en 2000. Aux deux repas, les grandes tablées favorisent la rencontre entre gens qui ne se connaissent pas. Bien sûr des groupes d'affinités se reforment, les conflits éventuels qui les opposent ne disparaissent pas mais les nouveaux s'installent dans les interstices entre ces groupes avec lesquels ils entrent, temporairement, en contact. La communication n'est pas toujours simple, les différences sociales et culturelles ne s'effacent pas mais « c'est un carrefour » (M. D.) et il règne une convivialité certaine à laquelle les produits de la viticulture locale contribuent assurément! À Pessac, l’idée du repas public lancée au début des années 1980 a reçu un accueil fort tiède : on espérait 500 participants, il en est venu 200, ce qui est dérisoire par rapport à la population et plus encore si on compare avec Créon. La participation n'a vraiment décollé - 700 à 800 participants, jusqu'à 1500 participants en 1996 pour la $100^{e}$ Rosière - qu'à partir du moment où le reste de la fête a été dépouillé d'une partie de sa rigidité formelle, ce qui répondait donc effectivement à une attente de la population. En dehors des grands repas collectifs, nombre de petites occasions sont aménagées, en amont et en aval de l'élection et de la fête, où le boire et le manger ensemble sont les ingrédients indispensables pour faire prendre la sauce du lien social. Ainsi, à Pessac, brochettes et entrecôtes grillées rassemblent les voisins de la Rosière en dehors de la fête elle-même. À Créon, on n'en finirait pas de recenser les différents «pots » qui réunissent des groupes restreints construits uniquement autour de la Rosière : les parents 
du Rosier invitent ceux de la Rosière, ensemble ils invitent le Conseil municipal, le bureau du comité des Fêtes, les voisins de la Rosière organisent une petite fête de quartier ou bien c'est elle qui les invite, etc.

D'autre part, comme les legs ont perdu toute valeur ${ }^{9}$ la participation des populations est sollicitée pour doter la Rosière. À Créon, cela prend la forme d'une collecte de fonds conduite par la Rosière et le Rosier de l'année et leurs amis, pendant un mois, dans toutes les maisons. C'est l'occasion de parler de la coutume, de rappeler que chacun peut participer au repas du dimanche, de faire entrer en somme, la personne et le principe de la fête dans chaque foyer. Cette quête laborieuse permet de relier à la Rosière, de manière très lâche et temporaire, un maximum de personnes. Plus solides sont les liens établis au sein des équipes de quête où se côtoient des groupes d'âges qui n'ont pas l'occasion de se fréquenter ${ }^{10}$. Ni La Brède, ni Pessa n’ont pour le moment, aménagé la collecte de fonds sur un mode aussi socialisant : à La Brède, une liste établie par la Rosière sur le modèle de la liste de mariage, est déposée chez un commerçant et chacun peut contribuer à un item de la liste. À Pessac et La Brède, sont vendus de petits bouquets de fleurs artificielles.

Cet éventail d'occasions sociales montre que de multiples passerelles sont aménagées entre les populations ancienne et récente : différents degrés de proximité avec la Rosière (personne et symbole) sont possibles de sorte qu'on n'est pas nécessairement soit « dedans » soit « hors » de la fête, qu'on peut passer sans trop d'effort d'un degré de participation à un autre. Ce système diversifié est très certainement un facteur essentiel d'intégration des nouveaux habitants dans et par la fête. L'augmentation substantielle et constante des bénéfices de la quête (8000 francs en 1988 ; 25000 francs en 1997) et le fait que, d'après les témoignages, les nouveaux habitants participent autant voire plus que les anciens, indiquent, sinon un investissement personnel dans la fête, du moins un intérêt certain pour sa perpétuation. Les néo-habitants entrent dans la fête autant pour s'insérer dans un tissu social, que pour s'approprier une image pittoresque de la ruralité facile à vivre qu'ils sont venus chercher et qu'ils contribuent à pérenniser.

Tenter d'évaluer l'impact à long terme de ces épisodes sur la communication entre anciens et nouveaux habitants serait un malentendu sur le sens et la fonction de la fête : elle doit être prise en compte pour elle-même, dans le temps qui lui est imparti, comme un phénomène en soi (Isambert, 1982). Elle n'est pas l'image exacte et permanente de la société mais un moment exceptionnel où les choses et les relations sont différentes du reste du temps. Il est cependant essentiel que ce moment spécial existe : "La fête de la Rosière peut être à Pessac ce que le 14 juillet est ailleurs » $\left(\mathrm{M} . \mathrm{R} .{ }^{11}\right)$.

Cela met en évidence que si les réseaux d'interconnaissance ne fonctionnent plus guère en amont de l'élection, une démarche volontariste les reconstruit en aval. Et, certaines des relations instaurées autour de la Rosière sont socialement efficaces à plus long terme. Les enquêtes font, en effet, apparaître que la Rosière a infiniment plus de chance qu'une autre jeune fille de son âge de trouver du travail et ceci grâce aux réseaux de relations et au regard porté sur elle par l'ensemble de la communauté. À Créon, sur les vingt-cinq dernières années, aucune Rosière, à deux exceptions près, n’a connu de chômage lors de son entrée sur le marché du travail ${ }^{12}$. Les exceptions confirment a contrario l'efficacité du système : les Rosières qui ont connu le chômage n'habitaient plus dans le rayon où elles étaient reconnues. Un emploi n'est-il pas dans le monde contemporain un facteur d'intégration équivalent à celui que représentait un bon mariage au siècle dernier? Si la coutume se perpétue et connaît même un certain regain n'est-ce pas parce que, dans une conjoncture difficile, elle conjugue des formes de solidarité à la fois anciennes et actuelles?

9. À l'exception de celui de Lesparre, en or.

10. En l'absence d'un lycée à proximité, après le collège, les jeunes se dispersent dans les divers lycées de la région. En outre, les Rosiers sont encore au collège quand les Rosières sont au lycée, voire à l'université.

11. Maire de Pessac depuis 1989.

12. Sur la période, le taux de chômage des jeunes filles est d'environ $25 \%$. 


\section{ASSURER LA COHÉSION SOCIALE LOCALE}

Est-ce à dire pour autant qu'il y ait consensus? De fait, les élus sont unanimes, jamais le principe de la fête n'a été remis en cause par les habitants, même si tout le monde ne participe pas. À Créon, nous avons compté de 600 à 800 personnes sur la place lors du couronnement, mais il existe probablement des gens qui n'y ont jamais assisté. En revanche, il est clair que supprimer la Rosière provoquerait une levée de boucliers qu'aucun maire n'envisage d'affronter. Les critiques portent plus sur la manière faire, plutôt que sur le principe de la fête. Élus et responsables sont conscients que si l'élection et la préparation de la fête réactivent les clivages politiques, culturels, sociaux et confessionnels, le temps de la fête est l'occasion d'une trêve qui permet à la communauté de se donner à elle-même l'image d'un ensemble uni, sinon homogène. Il serait inconvenant d'exprimer publiquement des désaccords : "Plus peut-être que le lieu de la paix sociale réelle, [ces fêtes] apparaissent comme le lieu de la négation des conflits » (Lautman, 1985, p. 36). Bien que la trêve soit brève sur l'année, la réactivation périodique lui confère une certaine forme de pérennité, en pointillés en somme. Toutefois, la dernière évolution remarquable en matière de restauration du lien social, laisse à penser qu'on attend désormais de la Rosière qu'elle soit la médiatrice d'une trêve de bien plus longue portée à la fois par sa durée et son importance pour la cohésion sociale.

Dès l'instauration des fêtes, les débats ont été vifs pour savoir si la Rosière était une fête laïque ou chrétienne et, par conséquent, s’il fallait ou non supprimer la messe. Celle-ci reste indispensable à l'heure actuelle : les institutions républicaines ont besoin du moule de la liturgie chrétienne pour donner une certaine sacralité aux événements qui marquent la cohésion sociale. Dès lors, des «bricolages » ont été réalisés pour les Rosières non croyantes mais appartenant à des familles de tradition catholique : elles suivent une sorte de formation accélérée sur la forme du rite et diverses personnes se chargent pendant la cérémonie de leur rappeler, par signe, ce qu'elles doivent faire. Mais cette question est passée au second plan depuis les années 1990 où il est apparu urgent de trouver un moyen d'intégrer les populations non chrétiennes, musulmanes en particulier. C'est donc une étape capitale pour la construction du lien social qui a été franchie en 1998 à Pessac avec l'élection, à l'unanimité, dès le premier tour, de la première Rosière musulmane et une messe œcuménique mémorable. L'élue militait à l'Association des travailleurs immigrés et souhaitait profiter de sa position pour faire remonter aux autorités les besoins des enfants de sa cité auxquels elle donnait des cours d'alphabétisation. On voit clairement ici que la fête reflète moins une réalité sociale effective - l'intégration des populations immigrées reste à faire - qu'un projet social dans lequel la Rosière, en tant que personne et symbole, devient médiatrice.

\section{RÉINSCRIRE LE TEMPS DANS LE TERRITOIRE}

Parallèlement, une certaine permanence est reconstruite pour pallier la rupture partielle du fil du temps produite par l'urbanisation. L'extension de la durée du rôle de Rosière - passé depuis les années 1980 d'une journée à un mandat d'un an - illustre la volonté d'instaurer une continuité. Par ailleurs, la profondeur temporelle de l'ancrage territorial n'étant désormais un donné que pour les familles de «souche », minoritaires à Pessac, c'est un construit symbolique présentifié dans l'espace qui lui est substitué par deux moyens : multiplication des commémorations, des hommages aux morts, aux anciens, d'une part et investissement d'un nombre croissant d'espaces publics, d'autre part. Jusque récemment, l'hommage aux morts se limitait au dépôt de gerbes sur la tombe des testateurs, celle de Montesquieu à La Brède et, depuis la Première Guerre mondiale, aux monuments aux morts. À Créon, on fleurit désormais aussi les tombes des anciens Rosiers et Rosières, de membres actifs du comité des Fêtes et du Conseil municipal l'année de leur décès. Les anciens sont, quant à eux, convoqués de plusieurs manières : visites aux maisons de retraite, invitation des anciennes Rosières au repas du dimanche et auto-commémorations périodiques 
$\left(90^{\mathrm{e}}\right.$ et $100^{\mathrm{e}}$ Rosières). N'est-il pas paradoxal de tant solliciter les morts et les vieux dans une fête de la jeunesse? Le paradoxe se dénoue si l'on considère le risque de dilution des identités dans l'aire d'influence de Bordeaux que l'urbanisation fait courir aux communes. En effet, plus que tout autres, les anciens incarnent la continuité entre passé rural et présent rurbain. De la même manière, les tombes matérialisent l'histoire locale dans l'espace, réifient le fil du temps toujours rompu par la mort, toujours reconstruit par la mémoire. On peut donc dire que la fête de la Rosière apparaît « comme un processus symbolique de lutte collective contre la mort et comme facteur d'identité locale » (Lautman, 1985, p. 32).

La focalisation de l'attention sur l'espace se manifeste, par ailleurs de deux manières différentes suivant la taille de la commune. Jusqu'aux années 1960, à Pessac, les mentions spatiales se résumaient, dans les scénarios de fête, à une liste des principales rues empruntées par le cortège et au schéma des déplacements dans l'hyper centre. Depuis, on s'ingénie à faire passer le cortège par un maximum de quartiers et de lieux signifiants. Plusieurs années de suite ${ }^{13}$, un plan de la ville a été inséré dans les programmes pour permettre aux nouveaux habitants de suivre le parcours du cortège. Dans le même temps, on s'efforce d'élire des jeunes filles venant des différents quartiers de la ville et tout particulièrement des nouveaux. À Créon, moins étendue, le renforcement du marquage territorial se traduit par l'investissement d'abord de la place publique puis de quasiment tous les espaces publics, surtout ceux de plein air qui accroissent la visibilité de la fête. Rotation des quartiers, multiplication des lieux publics impliqués et parcours sont des manières de marquer symboliquement l'espace public et par là de se l'approprier collectivement au travers de la Rosière (Brunet et al., 1993).

\section{Conclusion : la Rosière est-elle (encore) rurale?}

Face à toutes ces modifications engendrées par l'urbanisation, on peut effectivement se poser la question. L'idée que l'élection d'une Rosière est une tradition rurale repose sur plusieurs choses. On a vu que, dans un premier temps, l'intégration de villes-rosières dans une conurbation avait entraîné la disparition de la coutume qui ne s'est maintenue que dans les communes rurales. Aujourd'hui, ce processus qui semblait inéluctable est interrompu, provisoirement peut-être. Dans les communes en cours d'urbanisation, la coutume apparaît utile (nécessaire?) pour élaborer une certaine urbanité, c'est-à-dire une continuité identitaire symbolique efficace pour créer un sentiment d'appartenance susceptible de rassembler des groupes sociaux potentiellement conflictuels. En somme, c'est désormais parce qu'il y a une Rosière, une coutume ancienne, que la commune peut se considérer en continuité avec son identité rurale. "Rurale » s'entend alors moins par rapport à des activités agricoles que par la « charge de ruralité » que porte la Rosière et qui permet de maintenir un certain décalage, en dépit des évolutions, avec les valeurs et modes de vie citadins. On rappellera les principaux éléments de l'ancrage rural de la Rosière pour mettre en évidence la manière dont la ruralité est maintenant mise en forme.

Aussi loin qu'on puisse remonter, la charge de ruralité de la Rosière repose sur une association entre la figure féminine et des végétaux, son nom même est éloquent à ce titre. La forme actuelle de la coutume résulte de la christianisation, au Ve siècle, d'innombrables fêtes païennes - celtes et romaines notamment - liées à la célébration du printemps, au culte solaire, à des rites agraires et de fécondité (Frazer, 1922; Roubin, 1977). L'ensemble païen et chrétien forme l'arrière plan de référence aux testaments de La Brède et Pessac qui mêlent évocations de la Vierge ${ }^{14}$ et des champêtres Rosalia et Ruralia romaines où la vigne et le vin jouaient un rôle particulier : « La couronne sera composée de trois fleurs très communes dans nos champs de la Brède, savoir la Violette, symbole de la vertu simple et solitaire, mais dont l'odeur suave la décèle malgré elle, la Rose blanche des haies, symbole de l'innocence, le Bluet, symbole de la fidélité et même de

13. Jusqu'à ce qu'un plan de Pessac soit vendu en kiosque.

14. Elle aussi incarne la Rose. 
l'espérance. [...] La jeune fille tiendra dans ses mains un épi de blé ${ }^{15}$ et un pampre de vigne qu'après le couronnement elle offrira au plus ancien cultivateur propriétaire de la paroisse, si d'ailleurs il est digne de respect. Ce sera un symbole de l'hommage rendu à la vieillesse par la jeunesse et par la vertu au travail agricole ». La Rosière est donc explicitement, dès l'origine, érigée en incarnation à la fois de la ruralité et de la jeunesse. L'urbanisation a, bien entendu, induit certains aménagements, quand fleurs des champs, épis de blé et vieux cultivateurs sont devenus introuvables. On est passé aux fleurs artificielles, puis à nouveau aux fleurs fraîches, mais issues de l'horticulture comme les épis de blé qu'on ne cultive plus ici. Quant au vieux cultivateur, il a fallu se contenter de jardiniers mais gageons qu'une nouvelle interprétation des testaments permettra bientôt d'honorer les ouvriers agricoles marocains des grands châteaux de vin. Le pampre de vigne, toujours facile à trouver, reste le signe tangible qu'une certaine ruralité demeure, bien que spatialement circonscrite et minoritaire. La réduction drastique du végétal (associé au rural) au profit du minéral (associé à la ville) est compensée par une profusion de fleurs à toutes les occasions et dans tous les lieux marqués par la Rosière : depuis une vingtaine d'années, la multiplication des échanges de bouquets est spectaculaire au point que le budget pour les fleurs a explosé. Les végétaux (dont la rose que l'Élue incarne) conjoignent plusieurs niveaux de sens : symboles des valeurs et qualités que la Rosière doit personnifier et marqueurs de la ruralité dès l'instauration de la tradition; évocations d'un fond archaïque de fêtes où les éléments naturels ont une place particulière; symboles d'une offre de paix en situation de tension. Les végétaux sont donc au cœur de la quête identitaire en jeu dans le renouveau de la fête : faire l'identité implique ici de faire consensus en faisant campagne, mais une campagne de plus en plus idéalisée, esthétisée au fur et à mesure que le lien avec le passé rural devient plus ténu.

Aujourd'hui, donner à voir une « belle campagne »- belle sur les plans esthétique et moral - est un enjeu majeur pour la construction identitaire tant au niveau interne qu'externe. Il s'agit, d'une part, de revaloriser aux yeux des habitants leur commune et ses traditions par le regard, qu'on espère positif, des autres sur soi. Et, d'autre part, il s'agit également de promouvoir, en direction des touristes notamment, l'image de communes qui ont su conjoindre préservation d'une certaine ruralité et adaptation aux attentes urbaines en matière de campagne. La fête patrimonialisée devient alors un produit qui vend une certaine qualité de vie ${ }^{16}$ et peut figurer au nombre des aménités susceptibles de développer l'attractivité d'un lieu.

La Rosière - personne, fête et symbole - conjoint une image désuète de la campagne (traditions, végétaux, religiosité sont mis en avant plutôt que tracteurs, vache folle et pesticides) et une image idéale de modernité (femme émancipée, dynamisme, autonomie et non pas violence, chômage et marginalité). C'est-à-dire que, en dépit des nombreuses évolutions que la fête a connues, et celles qui se profilent, elle reste en décalage avec la réalité de son temps. Or, des plus anciens témoignages (Anonyme, 1888; Segalen, 1982) à nos jours (1968 en particulier), des observateurs ont souligné le même décalage, soit pour s'en réjouir, soit, le plus souvent, par agacement. En somme, depuis longtemps, sinon toujours, la Rosière est perçue comme quelque peu rétrograde. Ce décalage est indispensable pour que la coutume soit traitée comme une tradition - car une tradition, c'est toujours quelque chose qui date - et, par là, qu'elle puisse symboliser une certaine continuité du temps et de l'identité, et devenir à la fois, objet patrimonial et facteur d'intégration sociale, voire de développement culturel et économique.

15. Le testament, rédigé en gascon, dit «blat», seigle, mais, par proximité phonétique et pour sa charge symbolique on a retenu «blé » comme traduction.

16. Le risque est évidemment de folkloriser la fête. 


\section{Bibliographie}

AgulHon (M.), 1979. - Marianne au combat - L'imagerie et la symbolique républicaines de 1789 à 1880, Paris, Flammarion, tome 1, 251 p.

Anonyme, 1888 (?). - « La Rosière de La Brède », Non identifié, Chap. XX, p. 159-167.

Brunet (R.), FERRAs (R.), THÉRY (H.), 1993. - Les mots de la géographie : dictionnaire critique, Paris, GIP

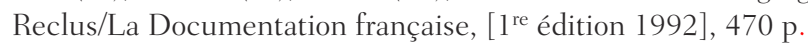

Darmian (J.-M.), 1986. - Créon, les Rosières du temps jadis, Artigues-près-Bordeaux, Imprimerie Delmas, $141 \mathrm{p}$.

Despouys (C.), 1996. - «Entretien avec la 100 Rosière de Pessac - 1996 », Bulletin de l'Institut Aquitain d'Études Sociales, Fêtes et identités, p. 87-111.

Frazer (J. G.), 1991. - The Golden Bough, London, Papermac, [1 éd.1922], p. 127-131.

IsAmbert (F.-A.), 1982. - « Le sens du sacré », dans Fête et religion populaire, Paris, Éditions de Minuit, $318 \mathrm{p}$.

LAUTMAn (F.), 1985. - «Fête traditionnelle et identité locale : Rêve?... ou recherche d'équilibre politique? », Terrain, n $^{\circ}$ 5, p. 29-36.

Roubin (L.), 1977. - «De l'églantier sauvage à la reine de mai », dans L'Autre et l'Ailleurs, hommage à R. Bastide, Paris, Berger Levrault, p. 190-201.

SEgalen (M.), 1982. - « Du village à la ville - La fête de la Rosière à Nanterre », Ethnologie Française, XII, p. 185-194.

Cet article a été reçu le 20 novembre 2006 et définitivement accepté le 20 juin 2007. 
